\title{
Large-scale structure and the Cardassian fluid
}

\author{
S. Fay ${ }^{1,2}$ and M. Amarzguioui ${ }^{3}$ \\ 1 School of Mathematical Science, Queen Mary, University of London, Mile End road, London E1 4NS, UK \\ e-mail: steph.fay@gmail.com \\ 2 Laboratoire Univers et Théories (LUTH), UMR 8102, Observatoire de Paris, 92195 Meudon Cedex, France \\ 3 Institute of Theoretical Astrophysics, University of Oslo, PO Box 1029 Blindern, 0315 Oslo, Norway \\ e-mail: morad@astro.uio.no
}

Received 3 February 2006 / Accepted 24 August 2006

ABSTRACT

\begin{abstract}
In this paper, we confront the predictions of the power law Cardassian model for the baryon power spectrum with the observations of the SDSS galaxy survey. We show that they fit only for very unusual values of the cold dark matter or baryon density parameters, the Hubble parameter or the spectral index of the initial power spectrum. Moreover, the best-fit Cardassian models turn out to be phantom models. If one wants to recover the usual values for these constants, as quoted by the WMAP team, the power law Cardassian model turns out to be indistinguishable from a $\Lambda \mathrm{CDM}$ model.
\end{abstract}

Key words. cosmology: theory - cosmological parameters - large-scale structure of Universe

\section{Introduction}

One of the greatest discoveries in cosmology in recent years is the accelerated expansion of the Universe. The first strong evidence that led to the conclusion that the expansion of the Universe appears to be accelerating, came in 1998 from observations of supernovae of type Ia (Riess et al. 1998; Perlmutter et al. 1999). Since then, more recent supernovae observations (Knop et al. 2003; Tonry et al. 2003; Riess et al. 2004) along with observations of the cosmic microwave background (Spergel et al. 2003) and large-scale structure (Tegmark et al. 2004) have strengthened this conclusion even further.

Now that the accelerated expansion of the Universe seems to have been established, the task facing cosmologists is to explain its origin. Several models have been proposed over the years that attempt to explain the dark energy that gives rise to the accelerated expansion. One such model is the power law Cardassian (Freese \& Lewis 2002). This is a model that has no additional energy components other than baryons and cold dark matter. However, motivated by brane world cosmology (Chung $\&$ Freese 2000), the Friedmann equation is modified by the presence of an additional energy term that is proportional to the cold dark matter density raised to a general power, i.e. $\rho_{\mathrm{m}}^{n}$. It is this term that gives rise to the accelerated expansion or dark energy. Indeed, it can be shown that the Hubble function can be written in the same form as ordinary general relativity with a dark energy fluid defined by a constant equation of state $p_{\mathrm{DE}} / \rho_{\mathrm{DE}}=n-1$, where $p_{\mathrm{DE}}$ and $\rho_{\mathrm{DE}}$ are respectively the pressure and density of this special form of dark energy (Avelino et al. 2003). Consequently, acceleration occurs for $n<2 / 3$ and the power law Cardassian model cannot be distinguished from such dark energy models by any observational tests based on the Hubble function only, such as the redshift-luminosity distance relation inferred from supernovae. However, this is no longer the case for tests based on large-scale structure data (Sandvik et al. 2004; Koivisto et al. 2005), since they probe more than just the background evolution. More general Cardassian models exist where the modification of the Friedmann equation cannot be written as a simple power in the matter density. Examples of such model are the Polytropic Cardassian Model (PC) and the Modified Polytropic Cardassian Model (MPC), which were proposed in Gondolo \& Freese (2003). However, for such models it is more difficult to make a connection to fundamental physics, hence rendering the model a purely phenomenological model with little physical motivation. We stress that we will consider only the original power law Cardassian model in this work. Furthermore, the model will be considered in the so-called fluid interpretation (Gondolo \& Freese 2003), which will be explained in Sect. 2. In the following, we will refer to the power law Cardassian model as a phantom Cardassian when $n<0$.

Constraints on Cardassian model imposed by the supernovae have been investigated extensively in several papers, e.g. (Sen \& Sen 2003; Wang et al. 2003; Frith 2004; Gong \& Duan 2004; Nesseris \& Perivolaropoulos 2004; Zhu et al. 2004; Bento et al. 2005, 2006; Lazkoz et al. 2005; Szydlowski et al. 2005, 2006). These studies show that the parameter space of the model is quite degenerate. In order to get better constraints one needs to consider additional cosmological tests. In Amarzguioui et al. (2005), the MPC model is constrained by comparing the matter power spectrum predicted by the model with that inferred from the SDSS large-scale structure data. The authors show that these data constrain the model severely. In fact, it is shown that $|n|$ has to be of order less than $10^{-5}$, which renders the model virtually indistinguishable from the $\Lambda \mathrm{CDM}$ model. In the analysis leading to this conclusion the density and Hubble parameters were fixed to the first-year values quoted by the WMAP team (Spergel et al. 2003), i.e. $\Omega_{\mathrm{m} 0}=0.224, \Omega_{\mathrm{b} 0}=0.046$ and $h=0.72$. Here, $\Omega_{\mathrm{m} 0}$ and $\Omega_{\mathrm{b} 0}$ denote the density parameters of dark matter and baryon respectively. In this paper we want to generalise this approach. We restrict ourselves to the power law Cardassian model, and perform a fit to the baryon power spectrum while allowing these cosmological parameters to take a range of constant values along with $n$. Furthermore, we allow also the 
spectral index $n_{\mathrm{s}}$ of the initial power spectrum to take a range of possible values rather than being just unity. This makes sense in view of the last WMAP results (Spergel et al. 2006) which give $n_{\mathrm{s}}=0.95 \pm 0.016$ compared with the first-year estimate of $0.99 \pm 0.04$. Does this added freedom allow the Cardassian model to deviate from the $\Lambda \mathrm{CDM}$ model? This is what we intend to answer in this paper.

We find that all the best-fit models have negative $n$, which in the terminology defined above means that they are phantom models. The models that are compatible with the data fall into two categories: $|n|$ is either so small that the model is indistinguishable from a $\Lambda \mathrm{CDM}$ model, or $n$ can have a non-negligible negative value. But in the latter case, one finds that either the cold dark matter density, the Hubble parameter or the spectral index must have very unusual values for the model to differ substantially from a $\Lambda \mathrm{CDM}$ model. Thus, if one wants to recover the usual values for these constants, as quoted by the WMAP team, the Cardassian model turns out to be indistinguishable from a $\Lambda$ CDM model with $|n|<10^{-5}$.

The structure of this paper is as follows: in Sect. 2 we present the Friedmann equation of the power law Cardassian model and look at how first order perturbations evolve in the fluid interpretation of this model. In Sect. 3 we discuss how to obtain the baryon power spectrum for the Cardassian model using the perturbed equations presented in the preceding section, and what initial condition to use for these. In Sect. 4 we present the statistics we need to fit the predicted power spectrum of the model with the observed. In Sect. 5, we fit the predictions of the Cardassian model with the SDSS (Tegmark et al. 2004) baryonic power spectrum by keeping three of the parameters $\Omega_{\mathrm{m} 0}, \Omega_{\mathrm{b} 0}, h, n_{\mathrm{s}}$ fixed to their WMAP concordance values and allowing the remaining one to take a range of possible values in addition to $n$.

Finally, in Sect. 6 we summarise and conclude.

\section{Field equations}

We assume the universe to be homogeneous, isotropic and flat. The metric can then be written in the usual form:

$\mathrm{d} s^{2}=-\mathrm{d} t^{2}+a(t)^{2}\left(\mathrm{~d} r^{2}+r^{2}\left(\mathrm{~d} \theta^{2}+\sin ^{2} \theta \mathrm{d} \phi^{2}\right)\right)$.

The modified Friedmann equation for the Cardassian model is

$H^{2}=\frac{8 \pi G}{3}\left(\rho_{\mathrm{m}}+\rho_{\mathrm{b}}+\rho_{\mathrm{c}}\right)$,

where $\rho_{\mathrm{m}}$ and $\rho_{\mathrm{b}}$ are respectively the CDM and baryonic matter densities and $\rho_{\mathrm{c}} \sim \rho_{\mathrm{m}}^{n}$. Note that we are looking at the ordinary Cardassian model and not the Modified Polytropic Cardassian Model (MPC) that was considered in Amarzguioui et al. (2005).

Energy conservation of pressureless matter implies that $\rho_{\mathrm{m}}, \rho_{\mathrm{b}} \sim(1+z)^{3}$ and hence, $\rho_{\mathrm{c}} \sim(1+z)^{3 n}$. This allows us to write the Friedmann equation as

$\left(\frac{H}{H_{0}}\right)^{2}=\left(\Omega_{\mathrm{m} 0}+\Omega_{\mathrm{b} 0}\right)(1+z)^{3}+\Omega_{\mathrm{c} 0}(1+z)^{3 n}$,

where we have defined the density parameters

$\Omega_{\mathrm{m} 0}=\frac{8 \pi G \rho_{\mathrm{m} 0}}{3 H_{0}^{2}}, \quad \Omega_{\mathrm{b} 0}=\frac{8 \pi G \rho_{\mathrm{b} 0}}{3 H_{0}^{2}}, \quad \Omega_{\mathrm{c} 0}=\frac{8 \pi G \rho_{\mathrm{c} 0}}{3 H_{0}^{2}}$.

In order to derive the matter power spectrum we need to go one step further than Eq. (3) which gives the evolution of the background. We need to consider first order perturbations. In order to do this, we follow the approach of Amarzguioui et al. (2005) and consider the fluid interpretation of the Cardassian model as introduced in Gondolo \& Freese (2003). In this approach, the dark matter term and the term that gives rise to dark energy are treated as two "components" of one single fluid - the Cardassian fluid. We write

$\rho=\rho_{\mathrm{m}}+\rho_{\mathrm{c}}=\rho_{\mathrm{m}}+\frac{\Omega_{\mathrm{c} 0}}{\Omega_{\mathrm{m} 0}} \rho_{\mathrm{m} 0}^{1-n} \rho_{\mathrm{m}}^{n}$.

Treating this as a perfect fluid and using energy-momentum conservation, we arrive at the usual continuity equation for the fluid $\rho$ :

$\dot{\rho}+3 H(\rho+p)=0$.

Substituting from Eq. (5) and using energy conservation of ordinary matter, the effective pressure of the Cardassian fluid can be written as

$p=p\left(\rho_{\mathrm{m}}\right)=\rho_{\mathrm{m}} \frac{\mathrm{d} \rho}{\mathrm{d} \rho_{\mathrm{m}}}-\rho$.

The sound speed and the effective equation of state for the Cardassian fluid can now be calculated easily. We get

$$
\begin{aligned}
c_{\mathrm{s}}^{2} & =\frac{\partial p}{\partial \rho}=\rho_{\mathrm{m}} \frac{\mathrm{d}^{2} \rho / \mathrm{d} \rho_{\mathrm{m}}^{2}}{\mathrm{~d} \rho / \mathrm{d} \rho_{\mathrm{m}}} \\
& =\frac{n(n-1)\left(\Omega_{\mathrm{m} 0}+\Omega_{\mathrm{b} 0}-1\right)(1+z)^{-3}}{n\left(\Omega_{\mathrm{m} 0}+\Omega_{\mathrm{b} 0}-1\right)(1+z)^{-3}-\Omega_{\mathrm{m} 0}(1+z)^{-3 n}}
\end{aligned}
$$

for the sound speed, and

$$
\begin{aligned}
\omega & =\rho_{\mathrm{m}} \frac{\mathrm{d} \rho / \mathrm{d} \rho_{\mathrm{m}}}{\rho}-1 \\
& =\frac{(n-1)\left(\Omega_{\mathrm{m} 0}+\Omega_{\mathrm{b} 0}-1\right)(1+z)^{-3}}{\left(\Omega_{\mathrm{m} 0}+\Omega_{\mathrm{b} 0}-1\right)(1+z)^{-3}-\Omega_{\mathrm{m} 0}(1+z)^{-3 n}}
\end{aligned}
$$

for the equation of state.

In the fluid interpretation, the model we're considering contains two fluids, namely the Cardassian fluid and the baryon fluid. The evolution of density perturbation in these fluids is given by a coupled set of second order differential equations. The detailed derivation of these equations can be found in Lyth $\&$ Stewart (1990) and Padmanabhan (1993). Setting the equation of state parameter and sound speed of baryons equal to zero, we can write these differential equations as

$$
\begin{aligned}
& \ddot{\delta}+H\left(2-3\left(2 w-c_{\mathrm{s}}^{2}\right)\right) \dot{\delta}-\left(\frac{3}{2} H^{2}\left(7 w-3 w^{2}-6 c_{\mathrm{s}}^{2}\right)-\left(\frac{c_{\mathrm{s}} k}{a}\right)^{2}\right) \delta \\
& -\frac{3}{2} H^{2}(1+w) \frac{\rho \delta+\rho_{\mathrm{b}} \delta_{\mathrm{b}}}{\rho+\rho_{\mathrm{b}}}=0
\end{aligned}
$$

and

$\ddot{\delta}_{\mathrm{b}}+2 H \dot{\delta}_{\mathrm{b}}-\frac{3}{2} H^{2} \frac{\rho \delta+\rho_{\mathrm{b}} \delta_{\mathrm{b}}}{\rho+\rho_{\mathrm{b}}}=0$,

where $\delta$ and $\delta_{\mathrm{b}}$ are the perturbations in the Cardassian and baryon fluids respectively. These equations are written with respect to cosmic time $t$. We want to solve them numerically, and in that respect it is more useful to write them in terms of a new time variable $u$ defined by

$$
\frac{\mathrm{d}}{\mathrm{d} t}=H \frac{\mathrm{d}}{\mathrm{d} u}
$$




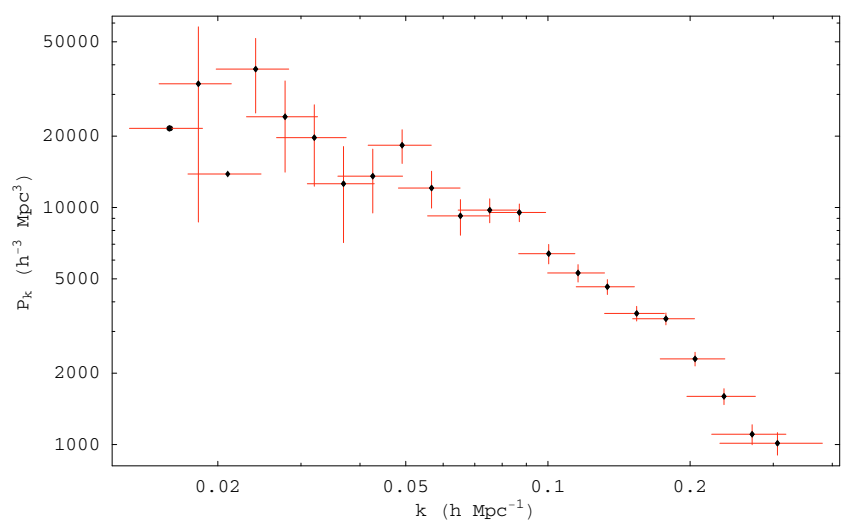

Fig. 1. The 22 data points of the SDSS power spectrum.

This implies that $a=\mathrm{e}^{u}=1 /(1+z)$ if we define the scale factor today as $a_{0}=1$. Using this time parameter and marking a derivative with respect to it with a prime, the differential equations take the form

$$
\begin{aligned}
H^{2} \delta^{\prime \prime}+ & \left(H^{2}\right)^{\prime} \frac{\delta^{\prime}}{2}+\left[2-3\left(2 \omega-c_{\mathrm{s}}^{2}\right)\right] H^{2} \delta^{\prime}-\frac{3}{2}\left(7 \omega-3 \omega^{2}\right. \\
& \left.-6 c_{\mathrm{s}}^{2}\right) H^{2} \delta+\frac{k^{2}}{\mathrm{e}^{u}} c_{\mathrm{s}}^{2} \delta-\frac{3}{2} H^{2}(1+\omega) \frac{\rho \delta+\rho_{\mathrm{b}} \delta_{\mathrm{b}}}{\rho+\rho_{\mathrm{b}}}=0 \\
H^{2} \delta_{\mathrm{b}}^{\prime \prime}+ & \left(H^{2}\right)^{\prime} \frac{\delta_{\mathrm{b}}^{\prime}}{2}-\frac{3}{2} H^{2} \frac{\rho \delta+\rho_{\mathrm{b}} \delta_{\mathrm{b}}}{\rho+\rho_{\mathrm{b}}}=0 .
\end{aligned}
$$

\section{Power spectrum}

In this work we will use the large-scale structure data of the SDSS team to constrain the Cardassian model. The galaxy power spectrum inferred from these data ${ }^{1}$ is plotted in Fig. 1.

The power spectrum of energy component $i$ can be written as $P_{i}(k) \sim \delta_{i}(k)^{2}$. Thus, we can use Eqs. (13) and (14) with the appropriate initial conditions to predict the power spectrum of the baryons and/or the Cardassian fluid today. Taking the arguments presented in Beça et al. (2003) into account, we will consider the power spectrum arising from the baryons only and not from the dark matter component of the Cardassian fluid.

To determine the initial conditions for the coupled system of differential Eqs. (13) and (14), we follow the approach of Sandvik et al. (2004) and Amarzguioui et al. (2005): we assume the evolution of the Cardassian and $\Lambda$ CDM models to be similar very early on, and use CMBFast (Seljak \& Zaldarriaga 1996) to calculate the matter transfer function at redshift $z=200$ arising from an initial spectrum with a general spectral index $n_{\mathrm{s}}$. This allows us to write the density perturbations at $z=200$ as

$\delta(k, z=200) \sim k^{n_{\mathrm{s}} / 2} T(z, k)$

where $T(z, k)$ is the transfer function obtained from CMBFast. Next, we use (13) and (14) to evolve the perturbations until today. The power spectrum of baryons today is then obtained as $P_{k} \sim \delta_{\mathrm{b}}(k, z=0)^{2}$.

\section{Statistics}

To fit the 22 SDSS data points for the power spectrum we will use a least square test $\chi^{2}$. It is defined by

$\chi^{2}=\sum_{i=1}^{22} \frac{\left(P(k, 0)_{i}^{\mathrm{obs}}-P(k, 0)_{i}^{\mathrm{th}} N\right)^{2}}{\sigma_{i}^{2}}$,

\footnotetext{
${ }^{1}$ http://www.hep.upenn. edu/ max/sdss.html
}

where $P(k, 0)$ is the power spectrum of the baryons today and the superscripts th and obs denote the theoretical and the observed spectra respectively. Furthermore, $\sigma_{i}$ are the $1 \sigma$ errors of the SDSS data and $N$ is a normalisation factor that allows us to adjust the amplitude of the theoretical power spectrum. We want to find the values of $N$ and the other cosmological parameters that minimise $\chi^{2}$. To simplify this procedure, we can define a new quantity $\bar{\chi}^{2}$ which minimises $\chi^{2}$ analytically with respect to the amplitude $N$. First, we define the following quantities:

$$
\begin{aligned}
& A=\sum_{i=1}^{22} \frac{\left(P(k, 0)_{i}^{\mathrm{th}}\right)^{2}}{\sigma_{i}^{2}} \\
& B=\sum_{i=1}^{22} \frac{P(k, 0)_{i}^{\mathrm{obs}} P(k, 0)_{i}^{\mathrm{th}}}{\sigma_{i}^{2}} \\
& C=\sum_{i=1}^{22} \frac{\left(P(k, 0)_{i}^{\mathrm{obs}}\right)^{2}}{\sigma_{i}^{2}}
\end{aligned}
$$

Substituting these into (16), we get the expression

$\chi^{2}=A N^{2}-2 B N+C$.

Treating this as a function of $N$ we find that its minimum is reached for $N=B / A$. We define the value of $\chi^{2}$ corresponding to this minimising $N$ as the new $\bar{\chi}^{2}$. Thus, we write

$\bar{\chi}^{2}=-\frac{B^{2}}{A}+C$.

This last expression defines a new $\chi^{2}$ analytically minimised with respect to $N$. This is the definition of $\chi^{2}$ that we will use to fit the data.

\section{Fit to the SDSS matter power spectrum}

In this section we perform the fitting of the theoretical Cardassian model to the empirical values of the SDSS matter power spectrum. The parameters which are constrained in the fitting are the Cardassian parameter $n$, the CDM density parameter $\Omega_{\mathrm{m} 0}$, the baryon density parameter $\Omega_{\mathrm{b} 0}$, the Hubble parameter $h$, and the spectral index $n_{\mathrm{s}}$. Ideally we would like to get a constraint on all of these parameters simultaneously, but the parameter space would be too large to handle in a simple $\chi^{2}$ test. We will therefore restrict ourselves to constraining pairs of these parameters with one parameter being $n$.

\subsection{Constraining the $n$ parameter only}

In this first subsection we mainly try to recover the results of Amarzguioui et al. (2005). In their analysis, the cosmological parameters were set equal to the values quoted by the WMAP team. However, they did not perform an explicit $\chi^{2}$ fitting to the data. They looked at the predicted power spectrum and noted that it deviates strongly from the SDSS data even for extremely small deviation from the $\Lambda$ CDM model. Repeating their analysis, but doing an explicit fit to the data, we set $\Omega_{\mathrm{m} 0}=0.224$ and $\Omega_{\mathrm{b} 0}=0.046$, implying $\Omega_{\mathrm{c} 0}=0.73$. Furthermore, we set the value of the Hubble parameter to $h=0.72$ and the spectral index to $n_{\mathrm{s}}=1$. Using Eq. (21), we find the minimal value for $\chi^{2}$ to be 27.08 when $n=-5.6 \times 10^{-7}$. With 22 data points and one free parameter, the $\chi^{2}$ per degree of freedom (d.o.f.) is thus $\chi_{\text {d.o.f. }}^{2}=1.28$. For $n=0$, corresponding to a cosmological constant, $\chi_{\text {d.o.f. }}^{2}=1.29$. These values are not very good but they are 


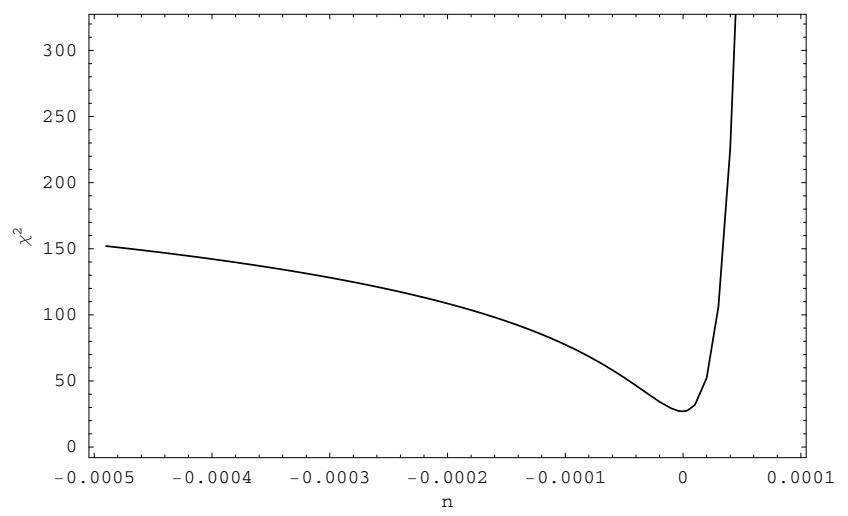

Fig. 2. $\chi^{2}$ as a function of $n$.

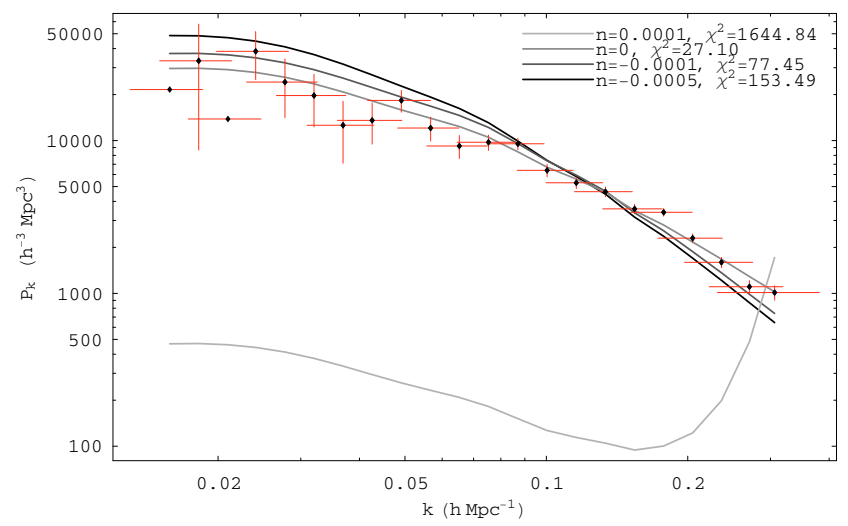

Fig. 3. Power spectrum predicted by the Cardassian model versus SDSS data for several values of $n$. The curves are such that they best fit the data. The power spectrum predicted with the positive value $n=0.0001$ is totally excluded by the data.

comparable to the values of $\chi_{\mathrm{d}}^{2}$ that were obtained with the 56 supernovae of Perlmutter (Perlmutter et al. 1999) published in 1999, and in works aiming to constrain quintessence models (Di Pietro \& Claeskens 2003). So maybe more precise data in the future will decrease the $\chi_{\text {d.o.f. }}^{2}$ we get in this section, just as was the case with the supernovae data. Figure 2 shows a plot of $\chi^{2}$ as a function of the parameter $n$.

We see clearly that the SDSS data do not allow for as large values of $n$ as the supernovae data: at $2 \sigma, n$ is limited to the range $n \in\left[-1.5 \times 10^{-5}, 8.9 \times 10^{-6}\right]$. They show that the Cardassian model must resemble a $\Lambda$ CDM model very closely to be in agreement with the large-scale structure observations and the WMAP values for the cosmological parameters. Figure 3 compares the predictions of the Cardassian model with the SDSS data and is in agreement with the results of Amarzguioui et al. (2005). Note that negative values for $n$ are not as strongly disfavoured as positive values. But still only a very small deviation from zero in the negative direction is allowed.

\subsection{Constraining $n$ and the CDM density parameter $\Omega_{m 0}$}

This section and the following ones generalise the work of Amarzguioui et al. (2005). We consider $n$ and $\Omega_{\mathrm{m} 0}$ to be free parameters and keep the other cosmological parameters fixed to $\Omega_{\mathrm{b} 0}=0.046, h=0.72$ and $n_{\mathrm{s}}=1$. The minimal value for $\chi^{2}$ is then 21.86, which gives us $\chi_{\text {d.o.f. }}^{2}=1.09$. This is obtained when $\Omega_{\mathrm{m} 0}=0.62$ and $n=-0.10$. In Fig. 4 we have plotted the $\chi^{2}$ along with the 1 and $2 \sigma$ confidence contours as functions

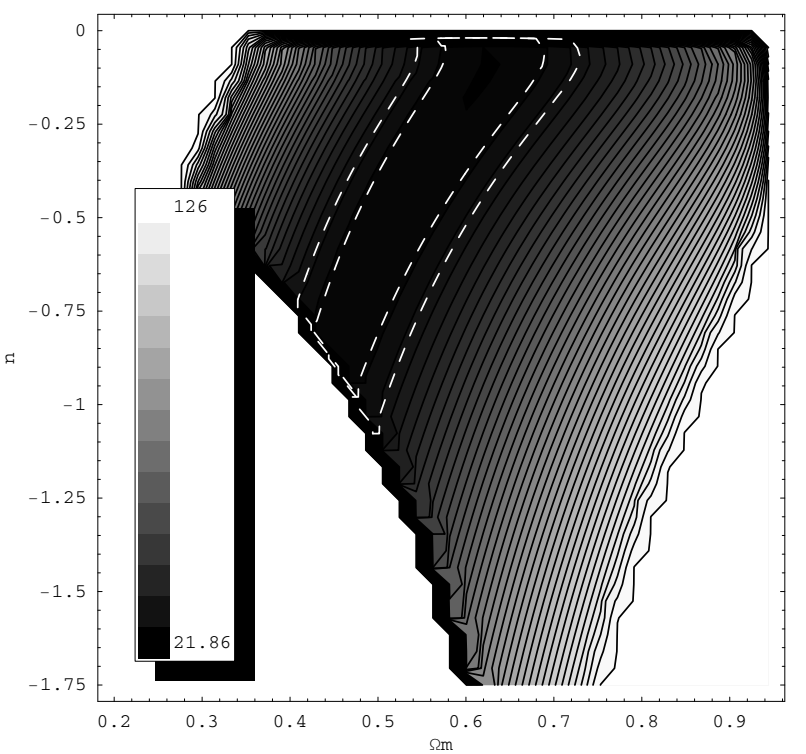

Fig. 4. $\chi^{2}$ and the 1 and $2 \sigma$ confidence contours (dashed lines) as functions of $n$ and $\Omega_{\mathrm{m} 0}$. We only present the values of $\chi^{2}$ smaller than 126 to clarify the colour of the plot.

of $n$ and $\Omega_{\mathrm{m} 0}$. In Fig. 5 one can find an enlargement of this figure for the area $\Omega_{\mathrm{m} 0}<0.4$. The 1 and $2 \sigma$ confidence levels for several ranges of values of $n$, in particular for the small $n$, are plotted in Fig. 6. At $2 \sigma$, the allowed ranges for the parameters are $n \in\left[-1.1,6.3 \times 10^{-6}\right]$ and $\Omega_{\mathrm{m} 0} \in[0.22,0.73]$. Thus, we see that positive values for $n$ are strongly disfavoured. But the data do allow for negative values up to order of unity when treating only $n$ and $\Omega_{\mathrm{m} 0}$ as free parameters.

In agreement with the previous section, for the fiducial value $\Omega_{\mathrm{m} 0}=0.224$, the Cardassian model fits the data only if $|n|<$ $O\left(10^{-5}\right)$, that is: it is indistinguishable from a $\Lambda$ CDM model. If we want the Cardassian model to be different from $\Lambda \mathrm{CDM}$, one has to consider some unusual values of $\Omega_{\mathrm{m} 0}$ like 0.6. Figure 7 shows predictions of the Cardassian model for the power spectrum versus SDSS data for some values of $\Omega_{\mathrm{m} 0}$ and $n$.

\subsection{Constraining $n$ and the baryon density parameter $\Omega_{b 0}$}

This time, we keep $\Omega_{\mathrm{c} 0}=0.73, h=0.72$ and $n_{\mathrm{s}}=1$ fixed, but treat $n$ and the baryon density parameter $\Omega_{\mathrm{b} 0}$ as free parameters when doing the fitting. The lowest $\chi^{2}$ value is now obtained for a negative value of $\Omega_{\mathrm{b} 0}$ (even with a prior on $\Omega_{\mathrm{b} 0}$ ) with $\chi^{2}=$ 21.55 . This is clearly an unphysical value. However degeneracy also allows positive values of $\Omega_{\mathrm{b} 0}$ with reasonable $\chi^{2}$, e.g. $\chi^{2}=$ 26.35 when $\Omega_{\mathrm{b} 0}=0.038$ and $n=-1.1 \times 10^{-5}$.

Figure 8 shows a plot of the $\chi^{2}$ and the 1 and $2 \sigma$ confidence contours as functions of $\Omega_{\mathrm{b} 0}$ and $n$. An enlargement of the confidence contours is shown in Fig. 9 for the most relevant area of the parameter space. The allowed ranges for the parameters at $2 \sigma$ are $n \in\left[-4.3 \times 10^{-5}, 5.3 \times 10^{-6}\right]$ and $\Omega_{\mathrm{b} 0} \in[0,0.048]$. Once again we find that the data constrains $n$ very strongly. Just as when we constrained $n$ alone, we find that the allowed range is smaller for positive than for negative values. But the constraint is still so strong that $n$ has to satisfy $|n|<10^{-5}$ in order for the Cardassian model to be in agreement with the SDSS data.

Figure 10 shows predictions of the Cardassian model for the power spectrum for some choices for $n$ and $\Omega_{\mathrm{b} 0}$ versus the SDSS data. We see from Fig. 9 that the data seem to prefer 

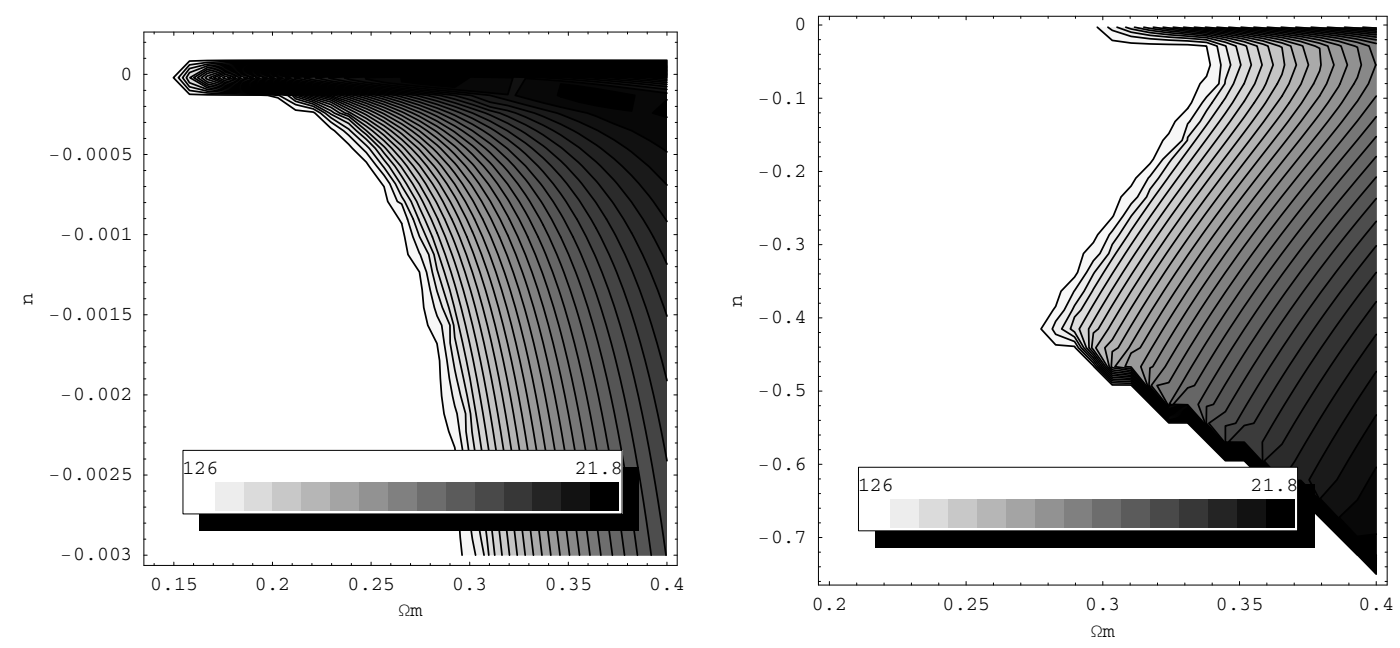

Fig. 5. Focus of Fig. 4 in the region of $\Omega_{\mathrm{m} 0}$ allowed by WMAP data.
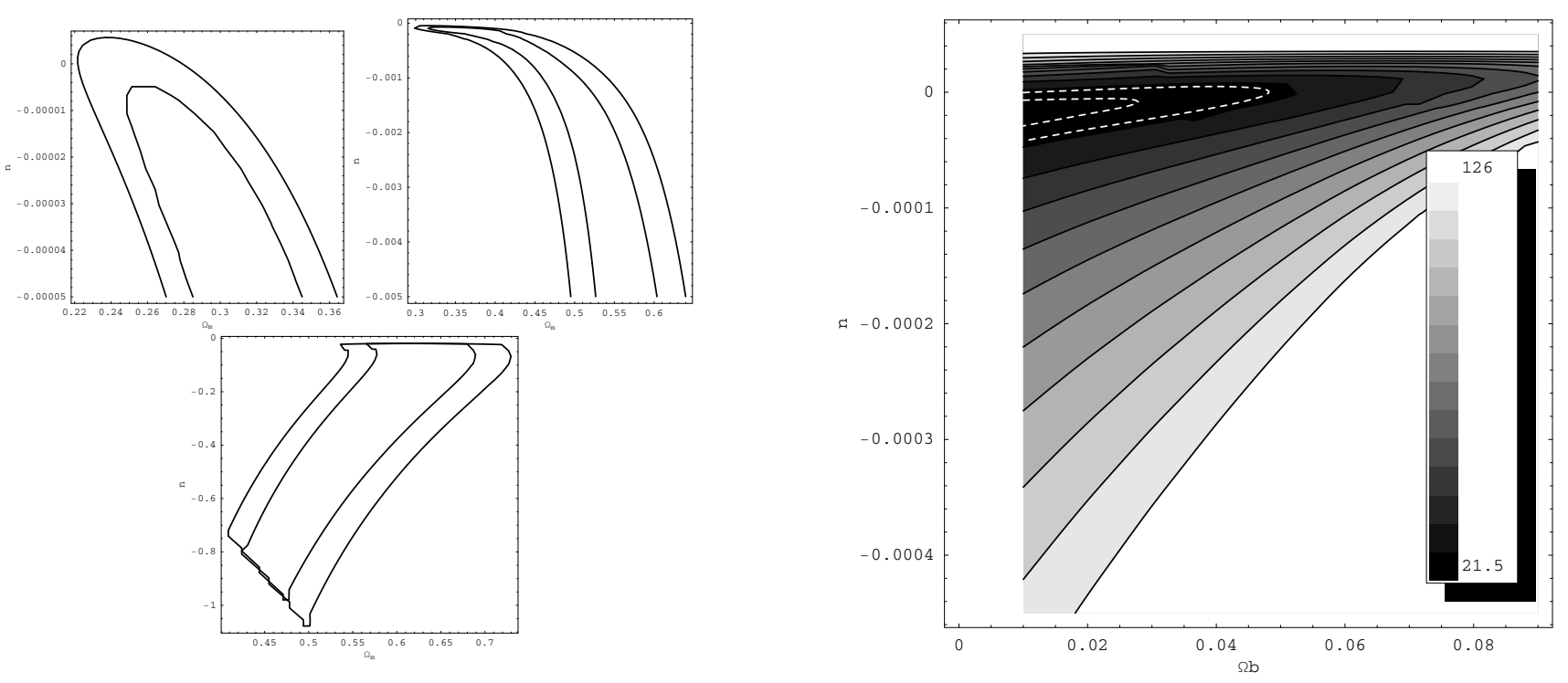

Fig. 6. 1 and $2 \sigma$ confidence contours for several ranges of $n$. Only very small values of $n$ are possible when $\Omega_{\mathrm{m} 0} \approx 0.224$, the value of the CDM density parameter in agreement with CMB data of WMAP.

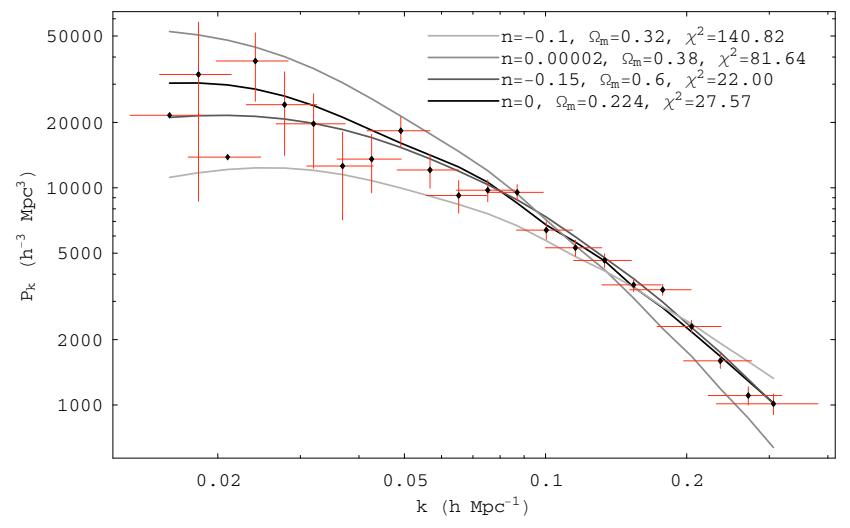

Fig. 7. Some power spectra predicted by the Cardassian model for several values of $\Omega_{\mathrm{m} 0}$ versus the SDSS data.

Fig. 8. $\chi^{2}$ and the 1 and $2 \sigma$ confidence contours (dashed lines) as functions of $\Omega_{\mathrm{b} 0}$ and $n$. Only the small value of $n$ allow to fit the SDSS data.

\subsection{Constraining $n$ and the Hubble parameter $h$}

Here, we treat $n$ and $h$ as free parameters in the fitting, and keep $\Omega_{\mathrm{m} 0}=0.224, \Omega_{\mathrm{b} 0}=0.046$ and $n_{\mathrm{s}}=1$ fixed. The best fit is now $\chi^{2}=21.02$ for $h=1.11$ and $n=-9.8 \times 10^{-5}$ with $\chi_{\text {d.o.f. }}^{2}=1.05$. Plots of the $\chi^{2}$ and the 1 and $2 \sigma$ confidence contours as functions of $h$ and $n$ can be found in Fig. 11. A couple of enlargements of the confidence contours are plotted in Fig. 12. The parameter ranges allowed at $2 \sigma$ are $n<1.8 \times 10^{-5}$ and $h>0.76$. We have not calculated the lower and upper limits for respectively $n$ and $h$ since they are beyond $h=1.40$ (and then below $n=-8 \times$ $10^{-4}$ ). Thus, the data appear to place a tight limit on positive values for $n$, but not on negative values, at least if one does not put a prior on $h$. Clearly, as the confidence contours show us, the SDSS data are not able to constrain the Hubble parameter very well.

Note that the HST Key Project (Freedman et al. 2001) quotes a value for the Hubble parameter of $h=0.72 \pm 0.8$, whereas the WMAP team quote a value of $h=0.72 \pm 0.05$ when using WMAP data alone. If we demand $h$ to be around 0.7 in order to be in agreement with these measurements, we find

values for $\Omega_{\mathrm{b} 0}$ that are smaller than the WMAP value. But still, the allowed values of $n$ are so small that it leaves the Cardassian model virtually indistinguishable from a $\Lambda$ CDM model. 


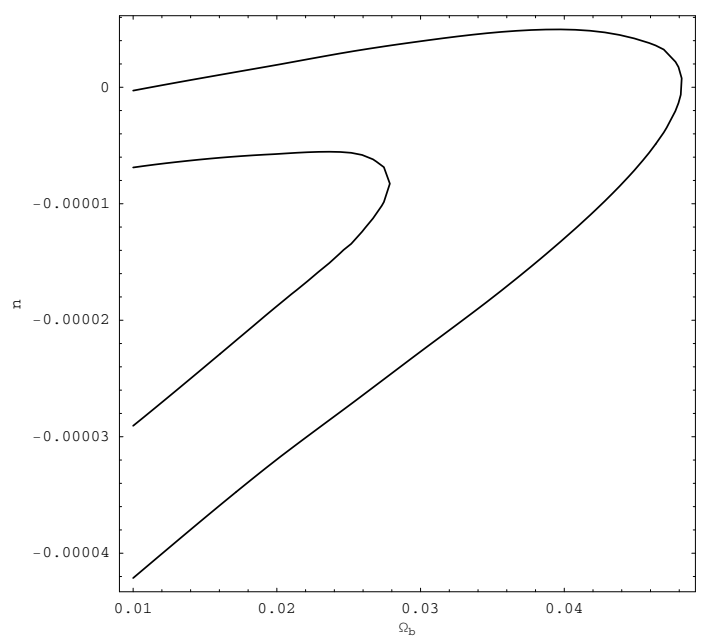

Fig. 9. The 1 and $2 \sigma$ confidence contours in the plane $\left(n, \Omega_{\mathrm{b} 0},\right)$.

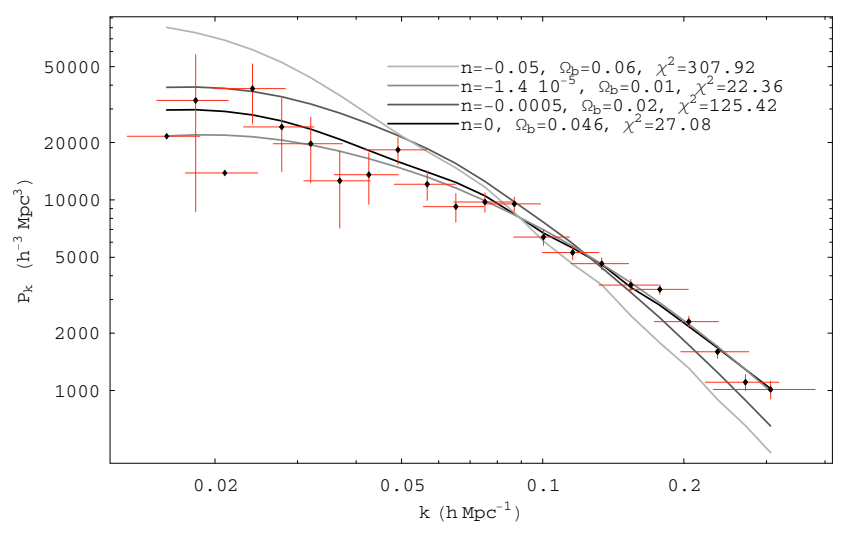

Fig. 10. Some power spectra predicted by the Cardassian model for several values of $\Omega_{\mathrm{b} 0}$ versus the SDSS data.

that $|n|$ must be less than $O\left(10^{-5}\right)$. Again, this implies that the Cardassian model is indistinguishable from the $\Lambda$ CDM model. The only way to make it substantially different is to accept values for $h$ larger than 1.0, which is quite unrealistic with respect to the measurements quoted above.

Figure 13 show plots of the power spectra predicted by the Cardassian model for some choices for the parameters $n$ and $h$.

\subsection{Constraining $n$ and the spectral index $n_{s}$}

In all the previous sections we have chosen the HarrisonZeldovich power spectrum $P=k^{n_{\mathrm{s}}}$ with $n_{\mathrm{s}}=1$ as the initial power spectrum. In this section we will consider $n_{\mathrm{s}}$ as a free parameter along with $n$. The other cosmological parameters are kept fixed with $\Omega_{\mathrm{b} 0}=0.046, \Omega_{\mathrm{m} 0}=0.224$ and $h=0.72$. The best fit is then $\chi^{2}=21.25$ with $\chi_{\text {d.o.f. }}^{2}=1.06$ when $n_{\mathrm{s}}=1.23$ and $n=-4.0 \times 10^{-5}$. The $\chi^{2}$ and the 1 and $2 \sigma$ confidence contours are plotted in Fig. 14 as functions of $n_{\mathrm{s}}$ and $n$. At $2 \sigma$ the allowed ranges for the parameters are $n \in\left[-0.3,4.2 \times 10^{-6}\right]$ and $n_{\mathrm{s}} \in[1,1.73]$. Just as we saw in the constraints in Sects. 5.2 and 5.4, positive values for $n$ are tightly constrained, whereas relatively large negative values are allowed.

Thus, it is evident that the SDSS data do not tightly constrain $n_{\mathrm{s}}$. Indeed, we find that for $n_{\mathrm{s}} \simeq 1.6$, large values of $n<0$ are in agreement with the SDSS data. Thus, if we accept such large values for the spectral index, it is then possible to construct a Cardassian model that differs considerably from $\Lambda \mathrm{CDM}$ and
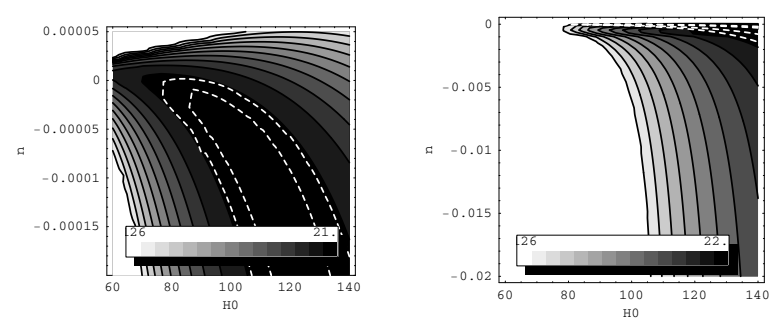

Fig. 11. $\chi^{2}$ and the 1 and $2 \sigma$ confidence contours (dotted line) as functions of $H_{0}$ and $n$.

still agrees with the SDSS data. However, such values of $n_{\mathrm{s}}$ are not compatible with the value measured by WMAP. For the special value $n_{\mathrm{s}}=1$, i.e. a Harrison-Zeldovich spectrum, we find that $|n|$ has to be less than $O\left(10^{-5}\right)$ in order to be in agreement with the data.

Once again, if we do not modify the usual HarrisonZeldovich power spectrum with $n_{\mathrm{s}}=1$, the Cardassian model is equivalent to a $\Lambda \mathrm{CDM}$ model. Figure 13 shows plots of the predicted power spectrum for the Cardassian model for several values of $n$ and $n_{\mathrm{s}}$ along with the SDSS data.

\section{Conclusions}

The purpose of this work has been to constrain the parameters of the power law Cardassian model in the fluid interpretation by using large scale structure data from the SDSS galaxy survey. We generalised the work of Amarzguioui et al. (2005) by treating additional cosmological parameters as free in addition to $n$ itself under a fitting to the galaxy power spectrum. In our analysis, we looked at five different fits to the SDSS galaxy power spectrum. In the first fit, repeating the analysis of Amarzguioui et al. (2005), we fixed the four cosmological parameters $\Omega_{\mathrm{m} 0}$ (CDM density), $\Omega_{\mathrm{b} 0}$ (baryon density), $h$ (Hubble parameter) and $n_{\mathrm{s}}$ (spectral index of the initial spectrum), and treated only the Cardassian parameter $n$ as a free parameter. Then one finds that $|n|$ has to be less that $10^{-5}$ so as to be in accordance with the data. A similar conclusion was reached by Koivisto et al. (2005) for the MPC model using the CMB power spectrum. These results are also similar to those of Sandvik et al. (2004), who showed that a generalised Chaplygin gas has to resemble the $\Lambda$ CDM model closely to avoid oscillations or blow ups of the matter power spectrum.

In the remaining four fits, we allowed one additional parameter to take values freely along with $n$, while keeping the remaining ones fixed to their WMAP best fit value. First, constraining $\Omega_{\mathrm{m} 0}$ simultaneously with $n$, we find that positive values for $|n|$ are tightly constrained, but not the negative. While the $2 \sigma$ upper limit for $\mathrm{n}$ is of order $10^{-6}$, the lower limit allows for negative values of order unity. But for such negative values the Universe would have to contain an unsual amount of cold dark matter. If we demand that the dark matter content be within the bounds given by WMAP, we again find that $|n|$ has to be so small that the model becomes indistinguishable from $\Lambda \mathrm{CDM}$.

Next, varying $\Omega_{\mathrm{b} 0}$ and $n$ freely, we again find that latter is strongly constrained both in the negative and positive directions. More specifically, $|n|$ has to be of order smaller than $10^{-5}$ to be in agreement with the data. Next, varying $h$ and $n$ freely, we again find that positive values for $n$ are tightly constrained, but negative values are much less constrained. However, it turns out $h$ has to be larger than $\sim 1.4$ in order for $n$ to be smaller than $-10^{-3}$. This is an unrealistically high value for the Hubble parameter. 

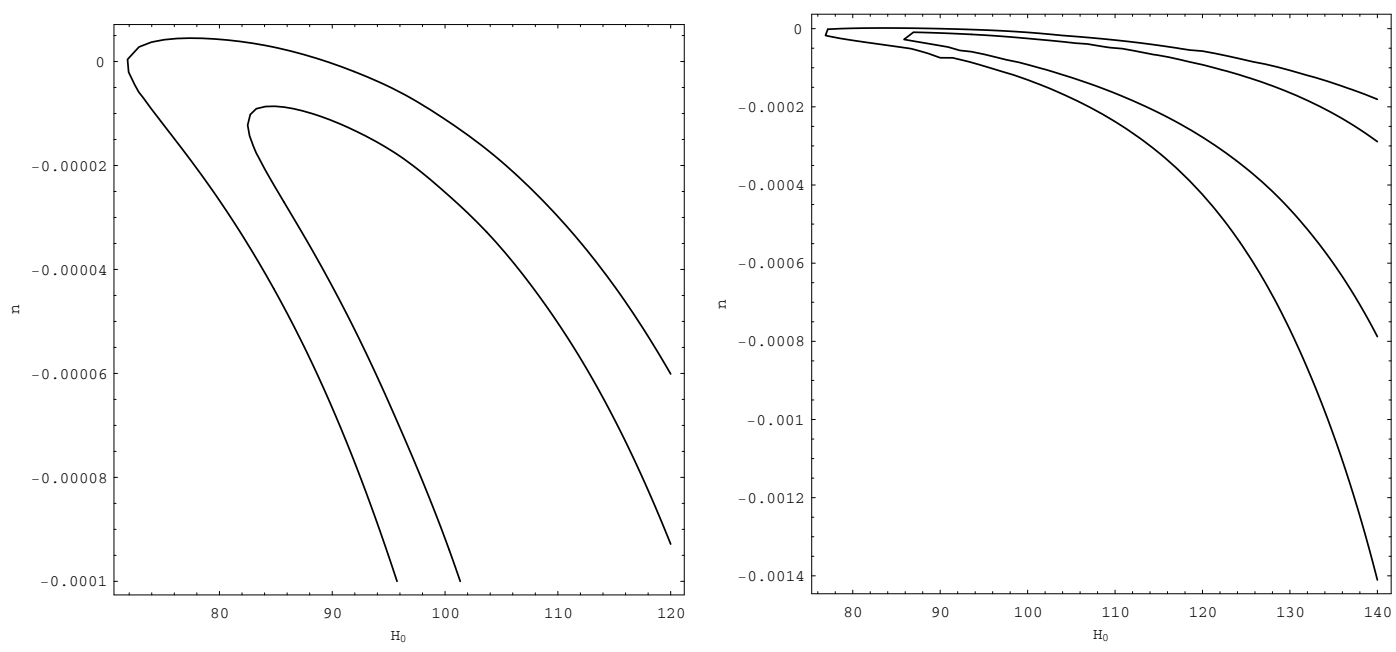

Fig. 12. 1 and $2 \sigma$ confidence contours as a function of $H_{0}$ and $n$.

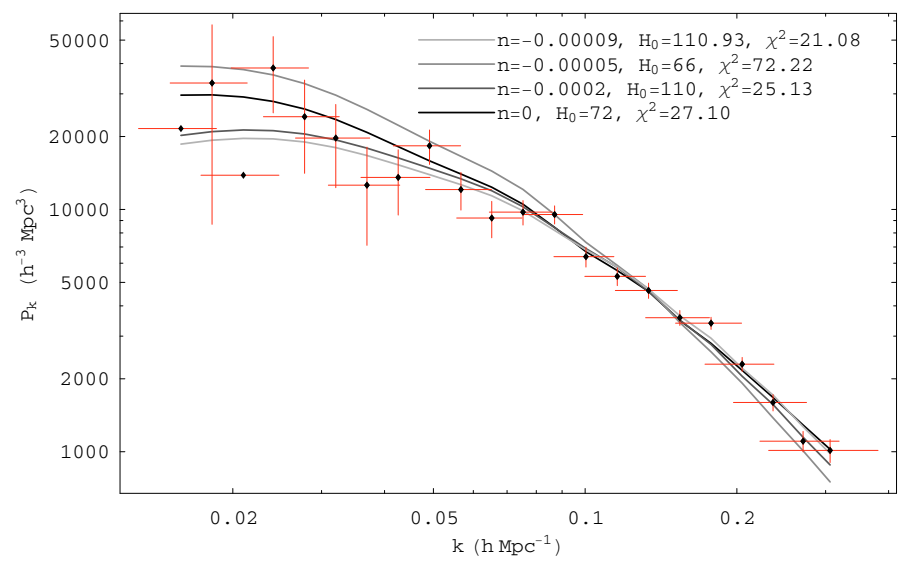

Fig. 13. Some power spectra predicted by the Cardassian model for several values of $H_{0}$ versus the SDSS data.
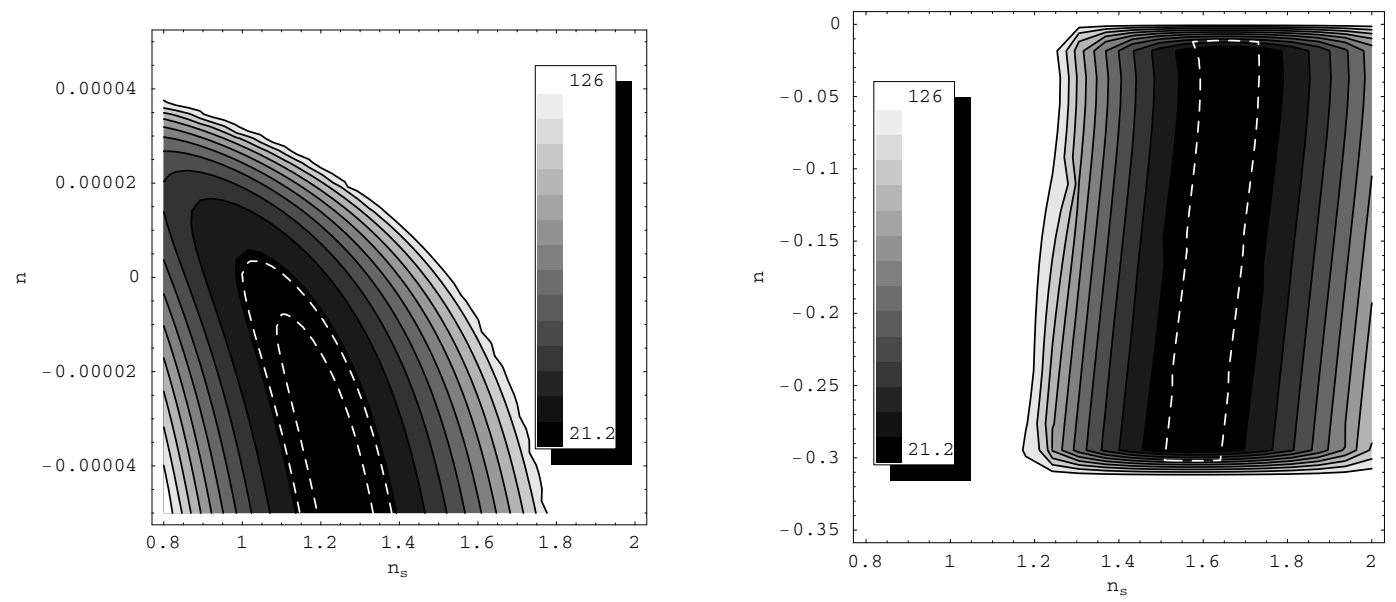

Fig. 14. $\chi^{2}$ and the 1 and $2 \sigma$ confidence contours (dotted lines) as functions of $n_{\mathrm{s}}$ and $n$. Note that in the second plot, the $1 \sigma$ confidence contour is not visible at this scale.

Demanding a more realistic value for $h$ will again render the Cardassian model indistinguishable from $\Lambda \mathrm{CDM}$.

Finally, allowing the spectral index $n_{\mathrm{s}}$ and $n$ to vary freely, we find the same behaviour as in the last fit. Positive values for $n$ are tightly constrained, while negative values are not. But adding a reasonable prior on $n_{\mathrm{s}}$ will restrict the allowed negative values severely. In fact, $n_{\mathrm{s}}$ has to be larger than $\sim 1.5$ in order for $n$ to be of order less than $-10^{-1}$ and still agree with the SDSS data.
A summary of our results showing the best fit models can be found in Table 1.

Hence, unless one is ready to accept very unusual values for the cosmological parameters considered here, the SDSS data force the power law Cardassian model to be virtually indistinguishable from an ordinary $\Lambda \mathrm{CDM}$ model. It thus seems difficult to unify dark energy and dark matter via the power law Cardassian model. 


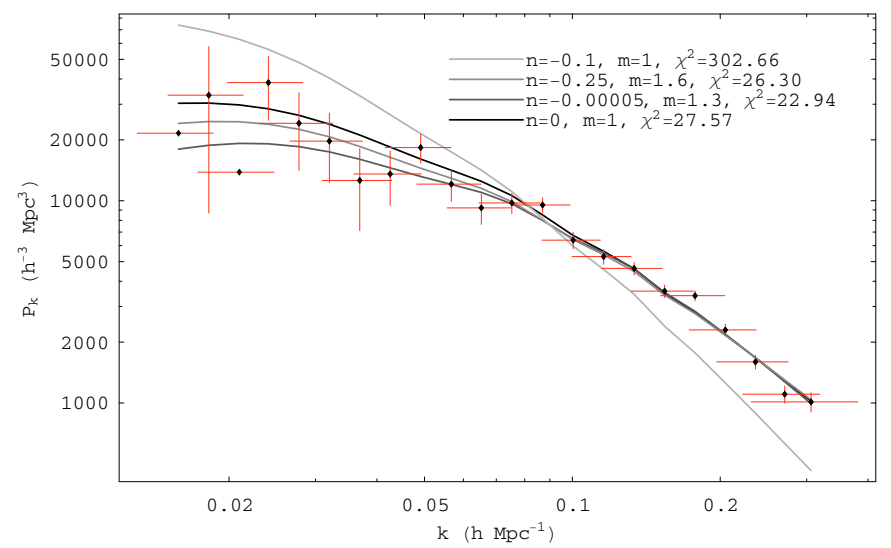

Fig. 15. Some power spectra predicted by the Cardassian model for several values of $n_{\mathrm{s}}$ and $n$ versus the SDSS data.

Table 1. Best values of the Cardassian parameters fitting the SDSS baryon spectrum.

\begin{tabular}{llcl}
\hline \hline Free parameters & Priors & $\chi_{\text {d.o.f. }}^{2}$ & Best fit \\
\hline$n$ & $n_{\mathrm{s}}=1, \Omega_{\mathrm{m} 0}=0.224$, & 1.28 & $n=-5.6 \times 10^{-7}$ \\
& $\Omega_{\mathrm{b} 0}=0.046, h=0.72$ & & \\
$n, \Omega_{\mathrm{m} 0}$ & $n_{\mathrm{s}}=1, \Omega_{\mathrm{b} 0}=0.046$, & 1.09 & $\Omega_{\mathrm{m} 0}=0.62$, \\
& $h=0.72$ & & $n=-0.1$ \\
$n, \Omega_{\mathrm{b} 0}$ & $n_{\mathrm{s}}=1, \Omega_{\mathrm{c} 0}=0.73$, & 1.08 & $\Omega_{\mathrm{b} 0}=-0.0022$, \\
$n, h$ & $h=0.72$ & & $n=-2.0 \times 10^{-5}$ \\
& $n_{\mathrm{s}}=1, \Omega_{\mathrm{m} 0}=0.224$, & 1.05 & $h=1.11$, \\
$n, n_{\mathrm{s}}$ & $\Omega_{\mathrm{b} 0}=0.046$ & & $n=-9.0 \times 10^{-5}$ \\
& $\Omega_{\mathrm{m} 0}=0.224$, & 1.06 & $n_{\mathrm{s}}=1.23$, \\
& $\Omega_{\mathrm{b} 0}=0.046, h=0.72$ & & $n=-4.0 \times 10^{-5}$ \\
\hline
\end{tabular}

Does this spell the end for the power law Cardassian modelat least in the fluid interpretation? A more general treatment is probably needed to conclude this absolutely. For example, one would ideally like to write a code like CMBFast for the Cardassian model instead of using the $\Lambda$ CDM model to generate the initial perturbations. However, this work confirms that the SDSS data look like a promising way to rule out this model. It shows that the galaxy power spectrum imposes much tighter constraints on the Cardassian model than the supernova data.

Finally, we wish to stress that we have only considered the Cardassian model in the fluid interpretation. If an alternative interpretation were to be found, where perturbative calculation could be performed, a similar analysis might very well produce a different result.

Acknowledgements. S.F. is supported by a Marie Curie Intra-European Fellowship Program of the Commission of the European Union (MEIF-CT-2005-515028) which is greatly appreciated. M.A. acknowledges support from the Norwegian Research Council through the project "Shedding Light on Dark Energy", grant 159637/V30. MA also wishes to thank $\emptyset y$ stein Elgarøy for helpful comments.

\section{References}

Amarzguioui, M., Elgarøy, Ø, \& Multamäki, T. 2005, JCAP, 0501, 008 Avelino, P. P., Beça, L. M., de Carvalho, J. P., Martins, C. J., \& Pinto, P. 2003, Phys. Rev. D, 67, 023511

Beça, L. M., Avelino, P. P., de Carvalho, J. P., \& Martins, C. J. 2003, Phys. Rev. D, 67, 101301

Bento, M. C., Bertolami, O., Santos, N. M., \& Sen, A. A. 2005, Phys. Rev. D, 71, 063501

Bento, M. C., et al. 2006, J. Phys. Conf. Ser., 33, 197

Chung, D. J. H., \& Freese, K. 2000, Phys. Rev. D, 61, 023511

Di Pietro, E., \& Claeskens, J.-F. 2003, MNRAS, 341, 4

Freedman, W. L., Madore, B. F., Gibson, B. K., et al. 2001, ApJ, 553, 47

Freese, K., \& Lewis, M. 2002, Phys. Lett. B, 540, 1

Frith, W. J. 2004, MNRAS, 348, 916

Gondolo, P., \& Freese, K. 2003, Phys. Rev. D, 68, 063509

Gong, Y.-G., \& Duan, C.-K. 2004, MNRAS, 352, 847

Knop, R. A., Aldering, G., Amanullah, R., et al. 2003, ApJ, 598, 102

Koivisto, T., et al. 2005, Phys. Rev. D, 71, 064027

Lazkoz, R., Nesseris, S., \& Perivolaropoulos, L. 2005, Exploring, JCAP, 0511, 010

Lyth, D. H., \& Stewart, E. D. 1990, ApJ, 361, 343

Nesseris, S., \& Perivolaropoulos, L. 2004, Phys. Rev. D, 70, 043531

Padmanabhan, T. 1993 (Cambridge: Cambridge University Press)

Perlmutter, S., Aldering, G., Goldhaber, G., et al. 1999, ApJ, 517, 565

Riess, A. G., Filippenko, A. V., Challis, P., et al. 1998, AJ, 116, 1009

Riess, A. G., Strolger, L.-G., Tonry, J., et al. 2004, ApJ, 607, 665

Sandvik, H., et al. 2004, Phys. Rev. D, 69, 123524

Seljak, U., \& Zaldarriaga, M. 1996, ApJ, 469, 437

Sen, S., \& Sen, A. A. 2003, ApJ, 588, 1

Spergel, D. N., Verde, L., Peiris, H. V., et al. 2003, ApJS, 148, 175

Spergel, D. N., et al. 2006, ApJ, submitted

Szydlowski, M., \& Czaja, W. 2005, Ann. Phys., 320, 251

Szydlowski, M., \& Godlowski, W. 2006, Phys. Lett. B, 633, 427

Tegmark, M., Strauss, M. A., Blanton, M. R., et al. 2004, Phys. Rev. D, 69, 103501

Tegmark M., Blanton, M. R., Strauss, M. A., et al. 2004, ApJ, 606, 702

Tonry, J. L., Schmidt, B. P., Barris, B., et al. 2003, ApJ, 594, 1

Wang, Y., Freese, K., Gondolo, P., \& Lewis, M. 2003, ApJ, 594, 25

Zhu, Z.-H., Fujimoto, M.-K., \& He, X.-T. 2004, ApJ, 603, 365 